ISSN electrónico: 2445-1355

DOI: http://dx.doi.org/10.14201/fj2019424150

\title{
BIOMARCADORES DE NEFROTOXICIDAD CAPACES DE IDENTIFICAR PACIENTES ONCOLÓGICOS EN RIESGO DE DESARROLLAR DAÑO RENAL
}

\section{Nephrotoxicity Biomarkers Able to Identify Oncological Patients at Risk of Developing Kidney Damage}

Laura PÉREZ-SÁNCHEZ ${ }^{1,2}$; Alfredo Ginés CASANOVA ${ }^{1,2,3}$; Ana Isabel MORALES ${ }^{1,2,3}$

1 Unidad de Toxicología, Dpto. Fisiología y Farmacología, Universidad de Salamanca, España.

2 Translational Research on Renal and Cardiovascular Diseases (TRECARD), Universidad de Salamanca, España.

${ }^{3}$ Instituto de Investigación Biomédica de Salamanca (IBSAL), España.

Correo-e: amorales@usal.es

RESUMEN: Cisplatino y carboplatino son dos fármacos muy usados en el tratamiento de tumores sólidos. Sin embargo, su eficacia está limitada por su nefrotoxicidad. Se ha demostrado que un 25-30 \% de los pacientes tratados con estos fármacos ha acabado desarrollando un daño renal agudo (DRA). En la actualidad, el diagnóstico del DRA se lleva a cabo mediante el uso de biomarcadores tales como creatinina plasmática y urea, pero resultan poco sensibles y específicos. El objetivo de este trabajo se centra en la búsqueda de biomarcadores urinarios capaces de predecir la aparición de un fallo renal, antes incluso de comenzar dicho tratamiento, siendo capaces de clasificar a los pacientes de acuerdo con su sensibilidad, predecir la evolución de su función renal y llevar a cabo un tratamiento más adaptado a cada paciente.

Un estudio clínico fue llevado a cabo con pacientes voluntarios del Servicio de Oncología del Hospital Universitario de Salamanca. Los biomarcadores estudiados fueron proteinuria, $\mathrm{N}$-acetil- $\beta$-glucosaminidasa (NAG), Lipocalina asociada a la gelatinada de neutrófilos (NGAL) y 
albúmina. Para la cuantificación de estos biomarcadores se utilizaron técnicas colorimétricas y la técnica ELISA.

Los resultaron mostraron que los niveles de los biomarcadores en el grupo Casos son significativamente superiores a los del grupo Control, tanto en el momento anterior al máximo daño renal, como en el momento basal, lo que nos permitiría saber cuáles son los pacientes que tendrían más probabilidad de sufrir un daño renal antes de iniciar el tratamiento. Dichos marcadores serían un gran avance en el tratamiento quimioterápico, ya que nos permitirían un tratamiento más preventivo y personalizado de cada paciente.

Palabras clave: cisplatino; daño renal agudo (DRA); predispodición adquirida; biomarcadores urinarios.

ABSTRACT: Cisplatin and carboplatin are very used drugs for the treatment of solid tumours. However, their efficacy is limited by their nephrotoxicity. It is known that $25-30 \%$ of the patients that have been exposed to these treatments will end up developing acute kidney injury (AKI). Nowadays, for the clinical diagnosis of AKI some biomarkers are used, such as plasma creatinine and urea, which are not very sensitive and specific. The aim of this work is focused on searching some urinary biomarkers able to predict the occurrence of a possible kidney damage even before starting the treatment, being able to classify the patients according to their severity, to predict the evolution of kidney function and to carry out a more adapted treatment for every patient.

A clinical study was carried out with voluntary patients from the Oncology Service of the University Hospital of Salamanca. The studied biomarkers were proteinuria, $\mathrm{N}$-acetil- $\beta$-glucosamidinase (NAG), neutrophil gelatinase-associated lipocalin (NGAL) and albumin. The quantification of these proteins in urine was performed by colorimetric methods and ELISA.

It was evidenced that the levels of the biomarkers in the basal time and in the one before the maximum kidney damage are practically the same, what indicates that these levels do not vary during the chemotherapy cycle. Moreover, they could indicate, before starting the treatment, that kidney damage is going to happen. These biomarkers would be a great advantage in determining, before undergoing the first chemotherapy cycle, whether a patient will suffer kidney complications.

Key words: cisplatin; acute kidney injury; acquired predisposition; urinary biomarkers. 
LAURA PÉREZ-SÁNCHEZ, ALFREDO GINÉS CASANOVA Y ANA ISABEL MORALES BIOMARCADORES DE NEFROTOXICIDAD CAPACES DE IDENTIFICAR PACIENTES ONCOLÓGICOS EN RIESGO DE DESARROLLAR DAÑO RENAL

\section{INTRODUCCIÓN}

El cisplatino y el carboplatino son dos fármacos muy utilizados en el tratamiento de tumores sólidos, destacando su uso en cáncer de ovario, cabeza y cuello, cérvix y pulmón (Rabik \& Dolan, 2007). Sus mecanismos de acción antineoplásica son muy similares. Al activarse intracelularmente, quedan libres dos valencias del ion platino, el cual forma dos enlaces estables con las bases nitrogenadas del ADN. El resultado es la alteración de la configuración tridimensional del material genético, la generación de errores de transcripción y la imposibilidad de que las cadenas se separen para la replicación (Basu \& Krishnamurthy, 2010). A pesar de su elevada efectividad en el ámbito clínico, su capacidad para causar nefrotoxicidad y ototoxicidad limita su utilización (Calderón-Ospina et al., 2012).

El cisplatino es el fármaco antineoplásico más nefrotóxico. Se ha demostrado que aproximadamente el 25-30\% de los pacientes tratados con él desarrollan un daño renal agudo (DRA) incluso después de una única dosis. Puede causar episodios leves, transitorios y auto-reparables de daño renal que pueden no tener consecuencias importantes para la salud. Sin embargo, la aparición de episodios acumulados puede dar lugar a consecuencias más perjudiciales. De hecho, ciclos repetidos de quimioterapia con cisplatino pueden conducir a fibrosis tubulointersticial crónica. Una pequeña fracción de estos pacientes necesitaría diálisis de por vida, y además, presentaría un alto riesgo de mortalidad a medio y largo plazo. Por lo tanto, la detección temprana de formas leves de DRA son metas no satisfechas para un mejor manejo de esta enfermedad (Vicente-Vicente et al., 2015).

Las principales manifestaciones clínicas del daño renal producido por cisplatino y carboplatino consisten en aumentos en las concentraciones séricas de creatinina y urea, y alteraciones electrolíticas. Entre las más frecuentes que se han descrito destaca la hipomagnesemia, consecuencia de la inhabilidad para la reabsorción tubular de magnesio que producen estos compuestos (Prada et al., 2011). Esta situación se suele ver acompañada de hipocalcemia e hipocaliemia. Dichos cambios en el transporte tubular de iones se deben, entre otras causas, a la alteración de la respiración celular producida por estos compuestos platinados (Launay-Vacher et al., 2008).

Hasta la fecha, el diagnóstico del DRA se lleva a cabo mediante unos marcadores indirectos de función renal, como la creatinina plasmática, que resulta poco sensible y específica, ya que se necesita al menos una disminución del $50 \%$ de la tasa de filtración glomerular (TFG) para poder detectarse un aumento de su concentración. Además, los niveles plasmáticos de este marcador dependen de otras variables, como la masa muscular, la función hepática o el volumen de distribución, que suelen variar en personas enfermas. Otro parámetro empleado 
es la urea plasmática, que tampoco es sensible y específica ya que puede sufrir una elevación como consecuencia de una lesión renal, pero también puede aumentar en ciertas condiciones como al seguir una dieta hiperproteica, tras una hemorragia gastrointestinal o durante un tratamiento con corticoides (Lombi et al., 2016).

A pesar de la potencial nefrotoxicidad que muestran estos fármacos, no todos los pacientes tratados con ellos acaban padeciendo DRA, sino que esto dependerá de la sensibilización que presente cada individuo a sufrir este trastorno. Esta predisposición a sufrir DRA puede ser genética, o se puede ir adquiriendo a lo largo de la vida y condicionará a que pacientes con el mismo tratamiento oncológico acaben o no desarrollando daño renal (Perazella, 2012). Esta última estaría relacionada con el desarrollo de un daño renal subclínico, que sería reparado por el organismo y no evolucionaría a DRA, pero sí aumentaría la susceptibilidad a padecerlo posteriormente. Este estado de sensibilización adquirida no es detectable mediante el uso de los marcadores de función renal que se utilizan actualmente (Vilander et al., 2015).

Por ello, se busca poder identificar precozmente a los pacientes con riesgo a desarrollar DRA, de forma más selectiva y específica que con los métodos actuales tras recibir un tratamiento quimioterápico (Seijas et al., 2014). Para ello, se están investigando biomarcadores de predisposición, que se definen como moléculas asociadas a patrones anatomopatológicos o a mecanismos patogénicos definidos, que detectan la sensibilidad a sufrir un daño renal y permiten clasificar a los pacientes en base a ella (Segarra-Medrano et al., 2012).

Así, el objetivo de este trabajo fue evaluar la utilidad de algunos de los biomarcadores de predisposición identificados por nuestro grupo de investigación en estudios animales, en pacientes oncológicos tratados con cisplatino o carboplatino, que faciliten la identificación de los pacientes con riesgo de desarrollar DRA en las fases iniciales de desarrollo, diagnosticarlo antes que las pruebas convencionales y poder pronosticar el desarrollo de la función renal del paciente e iniciar un tratamiento más adaptado.

\section{Materiales y mÉTOdos}

Se realizó un estudio clínico con pacientes voluntarios del Servicio de Oncología del Complejo Asistencial Universitario de Salamanca (CAUSA). Este cumplió con toda la legislación vigente en lo referente a los estudios clínicos y obtuvo la aprobación del Comité Ético de Investigación Clínica del CAUSA.

Tras llevar a cabo el reclutamiento de pacientes, se aplicaron los criterios de inclusión: que consistieron en ser mayor de 18 años, la aceptación voluntaria y firma de un consentimiento informado, y tener una función normal previa al 
tratamiento; y de exclusión: que no presentaran enfermedad o síntomas que por criterio del investigador pudiera interferir en los resultados del estudio. Tras la inclusión definitiva de los pacientes se llevó a cabo la recogida de sus muestras de orina y sangre. Dichas muestras se recogieron en dos tiempos: tiempo $0 \mathrm{~h}$ (antes de la primera administración del antineoplásico) y 72 h (tiempo teórico de máximo daño renal de los agentes platinados de acuerdo con la bibliografía (Salabarría, 2015)) en cada ciclo quimioterápico. Las muestras de sangre fueron enviadas al servicio de análisis clínicos, el cual nos facilitó los resultados de sus analíticas. Por otro lado, las muestras de orina fueron almacenadas en el Biobanco del CAUSA hasta su posterior utilización.

Se elaboró una base de datos informatizada donde se registraron los datos antropométricos, los factores de riesgo y los siguientes parámetros bioquímicos: creatinina y urea plasmáticas; y calcemia y magnesemia (marcadores sanguíneos clásicos empleados para diagnosticar un DRA causado por un antineoplásico platinado). En función de estos parámetros bioquímicos, los pacientes se distribuyeron en dos grupos: Casos (grupo de pacientes que sufrieron una alteración de al menos dos de los cuatro parámetros sanguíneos evaluados en algún momento de su tratamiento) y Controles (grupo de pacientes que no sufrieron esa alteración en al menos tres ciclos). Se seleccionaron, para cada paciente, la muestra urinaria basal y la muestra urinaria previa al momento de máximo daño renal.

Los biomarcadores cuantificados en dichas muestras de orina se presentan en la Tabla 1.

La cuantificación de proteinuria y de NAG se llevó a cabo mediante métodos colorimétricos. El fundamento de la colorimetría está basado en la medición de la absorción de radiación de la zona visible por sustancias coloreadas que se encuentren en una muestra, y su intensidad de coloración será directamente proporcional a sus concentraciones. Ya sea en el caso de la cuantificación de proteínas, mediante el método de Bradford, que está basado en la adición de un colorante. Como último paso, utilizamos la Ley de Lambert y Beer para la cuantificación de proteinuria y NAG, empleando una recta patrón de concentraciones conocidas de cada analito.

La NGAL y la albúmina se cuantificaron mediante la técnica ELISA (de sus siglas en inglés Enzyme Linked ImmunoSorbent Assay), en este caso, de tipo DAS (Double Antibody Sandwich). Esta técnica es un inmunoensayo que revela la presencia de antígenos $(\mathrm{Ag})$ o anticuerpos (Ac) mediante la formación de inmunocomplejos (complejos Ag-Ac) fijados a un soporte, los cuales tienen actividad enzimática. Mediante la adición de un sustrato específico que va a interactuar con ellos, dicha actividad enzimática generará un color cuantificable por espectrofotometría. 
TABLA 1. Fundamento teórico que justifica la detección de los biomarcadores urinarios analizados y su método de cuantificación.

\begin{tabular}{ccl}
\hline \multicolumn{1}{c}{ Biomarcador } & Método de cuantificación & \multicolumn{1}{c}{ Fundamento fisiopatológico } \\
\hline Proteinuria & Método de Bradford & $\begin{array}{l}\text { Se define como un exceso de } \\
\text { proteínas en orina. El aumento }\end{array}$ \\
& & $\begin{array}{l}\text { de su excreción se debe a una re- } \\
\text { absorción tubular disminuida de } \\
\text { las proteínas usualmente filtra- } \\
\text { das por el glomérulo (Montañés } \\
\text { et al., 2011). }\end{array}$ \\
\hline
\end{tabular}

\begin{tabular}{lll}
\hline N-acetil- & Método colorimétrico & La NAG se libera de los lisoso- \\
& mas presentes en el túbulo pro- \\
$\begin{array}{l}\beta \text {-D-glucosaminidasa } \\
\text { (NAG) }\end{array}$ & $\begin{array}{l}\text { ximal indicando daño celular. } \\
\text { Su presencia en orina permite }\end{array}$ \\
& identificar una lesión proximal \\
& (Manjón-Rodríguez, 2010). \\
\hline
\end{tabular}

Lipocalina asociada Técnica ELISA

a la gelatinasa de neutrófilos (NGAL) (kit 036 BioPorto®)
En el riñón, la NGAL se libera a las pocas horas tras una isquemia o tras un daño tubular tóxico, por lo que su medición en orina puede servir como marcador de daño temprano (Espinosa-Sevilla et al., 2013).

La albuminuria (presencia de albúmina en orina) marca un problema en la reabsorción tubular. Sin embargo, cuando la albuminuria es muy alta, esta puede deberse a una alteración en el proceso de filtración glomerular, por daño estructural o por una alteración en las cargas eléctricas de la membrana basal del glomérulo (Montañés et al., 2011).

En lo que respecta al análisis estadístico, todos los resultados se presentan como el promedio \pm error estándar de la media (EEM). El ajuste de resultados de cada grupo experimental a la distribución normal fue evaluado usando el test Kolmogorov-Smirnof. La comparación entre los dos grupos experimentales se realizó empleando un test Chi-cuadrado (para variables cualitativas) o un test $\mathrm{t}$ de Student o U de Mann-Whitney (para variables cuantitativas). 


\section{Resultados y discusión}

Los datos antropométricos y farmacológicos, así como la concentración de creatinina urinaria inicial de cada grupo de pacientes se presentan en la Tabla 2:

TABLA 2. Características iniciales, datos farmacológicos y concentración basal de creatinina urinaria de los pacientes incluidos en el estudio. Los valores se presentan en forma de Promedio \pm EEM. n.s.: No significativo.

\begin{tabular}{|l|c|c|c|}
\hline VARIABLE & $\begin{array}{c}\text { CASOS } \\
(\mathbf{n}=19)\end{array}$ & $\begin{array}{c}\text { CONTROLES } \\
(\mathbf{n}=17)\end{array}$ & p-valor \\
\hline Sexo (m/f) & $15 / 4$ & $9 / 8$ & n.s. \\
\hline Edad (años) & $60,7 \pm 1,8$ & $57,1 \pm 1,6$ & n.s. \\
\hline Peso (kg) & $78,9 \pm 3,2$ & $71,2 \pm 2,7$ & n.s. \\
\hline Altura (cm) & $166 \pm 2$ & $165 \pm 2$ & n.s. \\
\hline Índice de masa corporal (kg/m2) & $28,8 \pm 1,1$ & $26,4 \pm 1,3$ & n.s. \\
\hline Superficie corporal $(\mathbf{m})$ & $1,82 \pm 0,04$ & $1,76 \pm 0,03$ & n.s. \\
\hline $\begin{array}{l}\text { Pacientes tratados con cisplatino/ } \\
\text { carboplatino }\end{array}$ & $14 / 5$ & $9 / 8$ & n.s. \\
\hline Dosis de cisplatino $(\mathbf{m g})$ & $107 \pm 11$ & $99 \pm 13$ & n.s. \\
\hline Dosis de carboplatino $(\mathbf{m g})$ & $508 \pm 51$ & $625 \pm 41$ & n.s. \\
\hline Creatinina urinaria $(\mathbf{m g} / \mathrm{dL})$ & $81,9 \pm 14,2$ & $63,9 \pm 13,1$ & n.s. \\
\hline
\end{tabular}

A la vista de estos resultados, podemos comprobar que ambos grupos de estudio son estadísticamente semejantes, y con características antropométricas muy similares y homogéneas. Por ello, no podemos atribuir a ninguno de estos aspectos estudiados un mayor o menor riesgo a desarrollar un fallo renal.

Los resultados procedentes del análisis cuantitativo de biomarcadores en las muestras de orina se presentan en la Figura 1.

A la vista de los resultados, las cuatro gráficas nos muestran que los niveles urinarios de cada uno de los biomarcadores estudiados son superiores en el grupo Casos que en el grupo Controles. Atendiendo al momento basal y al pre-daño, comprobamos que las concentraciones de cada biomarcador son similares dentro de cada grupo.

La finalidad de este estudio es comprobar si hay algún biomarcador en los pacientes susceptibles que demuestre la posibilidad de desarrollar DRA antes que los biomarcadores utilizados actualmente en la práctica clínica. De aquí la 
FIgURA 1. Biomarcadores urinarios evaluados en las muestras de los pacientes oncológicos. NAG: N-Acetil-beta-D-glucosaminidasa; NGAL: Lipocalina asociada a la gelatinasa de neutrófilos. Los datos se expresan en forma de promedio \pm EEM. $* \mathrm{p}<0.05 ; * \mathrm{p}<0.01$ vs grupos Controles.

\section{Proteínas totales}

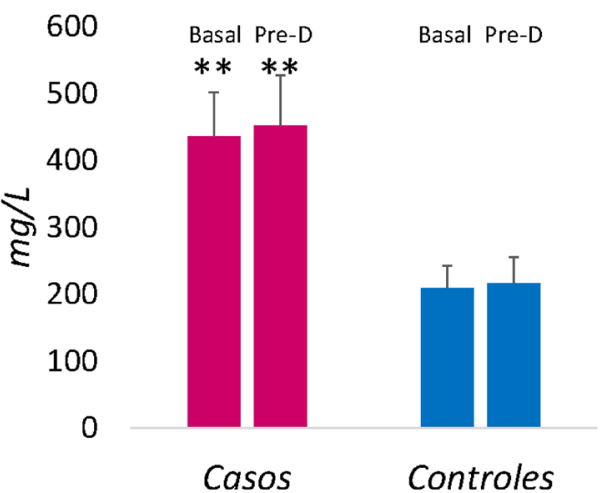

NGAL

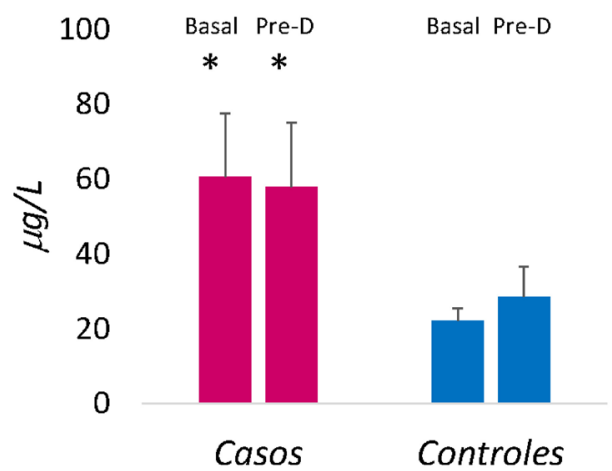

NAG

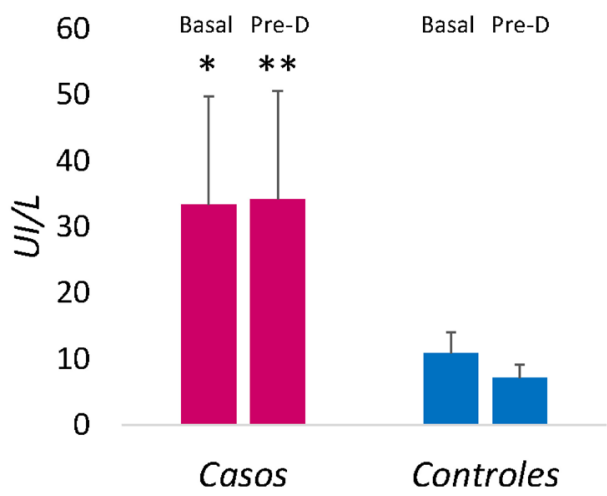

Albúmina

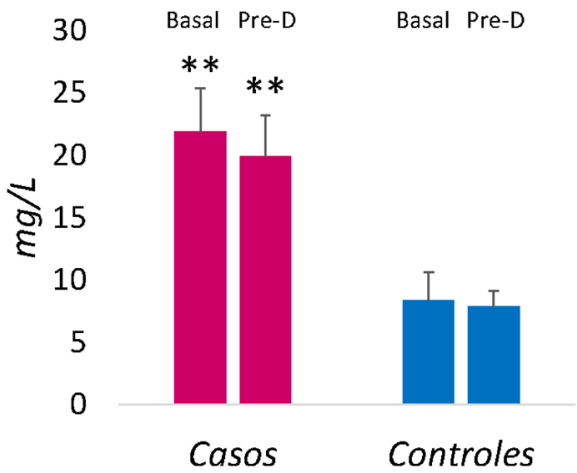

importancia de la selección de una muestra basal de cada paciente, para poder comprobar que los niveles de los biomarcadores eran ya superiores antes de empezar con el tratamiento quimioterápico.

Observando las gráficas, los pacientes del grupo Casos poseen niveles de los cuatro biomarcadores significativamente superiores a los del grupo Controles, por lo que podemos sospechar que pacientes con altas concentraciones de estos biomarcadores acabarán desarrollando DRA. 
Dentro de cada grupo, los niveles de biomarcadores de las muestras previas al daño son muy similares a las muestras basales, lo que quiere decir que se mantienen prácticamente estables a lo largo de los ciclos quimioterápicos. Por la misma razón, en el grupo de los Casos sabemos que los pacientes susceptibles a desarrollar DRA están, desde antes de iniciar el tratamiento, sensibilizados a sufrir complicaciones renales ante la administración de fármacos nefrotóxicos. Esto supone una ventaja, ya que nos indica que se podrá evaluar el grado de predisposición en cualquier momento, incluso antes de iniciar el tratamiento.

La justificación del aumento que se observa en la excreción urinaria de estos biomarcadores cuando va a desarrollarse DRA puede deberse, como se hace referencia en el apartado de introducción, a que los agentes platinados desencadenan procesos de apoptosis y necrosis celular y afectan al funcionamiento del epitelio tubular, lo que provocará un defecto en sus mecanismos de absorción.

Tras estos resultados, un objetivo futuro sería el diseño de un test capaz de detectar si un paciente es susceptible a padecer DRA. Esto conllevaría un gran avance en la práctica clínica, ya que mediante una muestra de orina podríamos estratificar a los pacientes en función de su riesgo, y asíllevar a cabo un tratamiento personalizado a cada paciente para disminuir las complicaciones renales, tales como monitorización de la volemia, de la hidratación, utilización de nefroprotectores, reducción de dosis, sustitución de antineoplásicos, etc.

\section{Conclusión}

Se confirma que los biomarcadores urinario proteínas totales, NAG, NGAL y albúmina se excretan de manera significativamente superior en los pacientes oncológicos que posteriormente van a sufrir un DRA. Por tanto, este estudio es un buen punto de partida para avanzar en el desarrollo de nuevas herramientas aplicadas al tratamiento preventivo y personalizado de pacientes oncológicos, como test rápidos que en contacto con orina predigan si el paciente es susceptible a desarrollar DRA, siendo de gran trascendencia clínica respecto a pronóstico y costes sanitarios.

\section{Bibliografía}

Basu A, Krishnamurthy S. Cellular Responses to Cisplatin-Induced DNA Damage. J Nucleic Acids. 2010; 2010:1-16.

Calderón-Ospina CA, Guzmán-Ramírez GM, Sarmiento-Monroy JC, Gómez-Angulo DL, Joya-Higuera AY, Ríos-Barajas LF et al. Nefrotoxicidad inducida por medicamentos. Rev. Médicas UIS. 2012; 24(1):65-85. 
Espinosa-Sevilla A, Amezcua-Macías AI, Ruiz-Palacios PC, Rodríguez-Weber F, DíazGreene E. Nuevos marcadores de lesión renal aguda en el enfermo grave. Med Interna México. 2013; 29(5):513-17.

Launay-Vacher V, Rey J-B, Isnard-Bagnis C, Deray G, Daouphars M. Prevention of cisplatin nephrotoxicity: state of the art and recommendations from the European Society of Clinical Pharmacy Special Interest Group on Cancer Care. Cancer Chemother Pharmacol. 2008; 61(6):903-9.

Lombi F, Muryan A, Canzonieri R, Trimarchi H. Biomarcadores en la lesión renal aguda: ¿paradigma o evidencia?. Nefrología. 2016; 36(4):339-46.

Manjón-Rodríguez MD. Estudio de N-acetil-B2-glucosaminidasa y otros marcadores de daño renal en nefroangioesclerosis hipertensiva. Editorial de la Universidad de Granada; 2010.

Montañés Bermúdez R, Gracia García S, Pérez Surribas D, Martínez Castelao A, Bover Sanjuán J. Documento de Consenso. Recomendaciones sobre la valoración de la proteinuria en el diagnóstico y seguimiento de la enfermedad renal crónica. Nefrol Madr. 2011; 31(3):331-45.

Perazella MA. Onco-Nephrology: Renal Toxicities of Chemotherapeutic Agents. Clin J Am Soc Nephrol. 2012; 7(10):1713-21.

Prada, M, Gastelbondo, R, Garcés, S. Nefrotoxicidad por Quimioterapia. Nefrol Pediátrica. 2011; 11(3):136-44.

Rabik CA, Dolan ME. Molecular mechanisms of resistance and toxicity associated with platinating agents. Cancer Treat Rev. 2007; 33(1):9-23.

Salabarría, J. Laboratorio clínico y función renal. 2015; 1-30.

Segarra-Medrano A, Carnicer-Cáceres C, Arbós-Via MA, Quiles-Pérez MT, AgrazPamplona I, Ostos-Roldán E. Biomarcadores en el síndrome nefrótico: algunos pasos más en el largo camino. Nefrol Madr. 2012; 32(5):558-72.

Seijas M, Baccino C, Nin N, Lorente JA. Definition and biomarkers of acute renal damage: New perspectives. Med Intensiva Engl Ed. 2014; 38(6):376-85.

Vicente-Vicente L, Sánchez-Juanes F, García-Sánchez O, Blanco-Gozalo V, Pescador M, Sevilla MA et al. Sub-nephrotoxic cisplatin sensitizes rats to acute renal failure and increases urinary excretion of fumarylacetoacetase. Toxicol Lett. 2015; 234(2):99-109.

Vilander LM, Kaunisto MA, Pettilä V. Genetic predisposition to acute kidney injury - a systematic review. BMC Nephrol. 2015; 16(1):197. 